\title{
Cambios Faciales y su Estabilidad en el Tiempo en Pacientes con Fisura Labiopalatina no Sindrómica Sometidos a Distracción Osteogénica Maxilar con Distractor Externo Rígido. Revisión Narrativa
}

\author{
Facial Changes and their Stability over Time in Non-Syndromic \\ Cleft Lip and Palate Patients Undergoing Maxillary Distraction \\ Osteogenesis Through Rigid External Distractor. Narrative Review
}

\author{
María Trinidad Guerrero Lehuede; Vicente Traub Valdés; María Ignacia Zursiedel Puentes; \\ Cristián Álamos Samur; Alfredo Noguera Pantoja; Carolina Gutiérrez Meliz \& Pedro Solé Ventura
}

GUERRERO, L. M. T.; TRAUB, V. V.; ZURSIEDEL, P. M. I.; ÁLAMOS, S. C.; NOGUERA, P. A.; GUTIÉRREZ, M. C. \& SOLÉ, V. P. Cambios faciales y su estabilidad en el tiempo en pacientes con fisura labiopalatina no sindrómica sometidos a distracción osteogénica maxilar con distractor externo rígido. Revisión narrativa. Int. J. Odontostomat., 13(2):123-131, 2019.

RESUMEN: Las fisuras labiopalatinas corresponden a las malformaciones de cabeza y cuello de mayor prevalencia a nivel mundial; dentro de las cuales, aquellas que no están asociadas a síndrome son las más frecuentes. Los pacientes que sufren estas malformaciones presentan en muchos casos hipoplasia maxilar. La distracción osteogénica maxilar mediante distractor externo rígido constituye una alternativa de tratamiento para la corrección de esta deficiencia esqueletal. Describir los cambios faciales y su estabilidad en el tiempo, en pacientes con fisura labio palatina no sindrómica sometidos a distracción osteogénica maxilar con distractor externo rígido. Se realizó una búsqueda estratégica en las bases de datos PubMed, Epistemonikos, EBSCO, BEIC y The Cochrane Library a través de las palabras clave cleft palate; cleft lip and palate; distraction osteogenesis; osteodistraction; callotasis; callotases; callus distraction, maxillary hypoplasia; midface hypoplasia; hypoplastic maxilla; maxillary deficiency; retromaxilla y maxillary retrognatism; con los términos booleanos AND y OR. Se seleccionaron 20 artículos: 2 revisiones sistemáticas, 3 ensayos clínicos, 14 estudios observacionales descriptivos y 1 estudio observacional analítico. La distracción osteogénica maxilar con distractor externo rígido corresponde a una alternativa efectiva en el tratamiento de la retrusión del tercio medio en pacientes con fisura labiopalatina no sindrómica. Esta técnica, sin embargo, no consigue cambios completamente estables, existiendo múltiples factores relacionados con su recidiva.

PALABRAS CLAVE: distracción osteogénica, fisura labiopalatina, osteotomía Le Fort, cirugía ortognática.

\section{INTRODUCCIÓN}

La fisura labiopalatina (FLP) corresponde a una deformidad congénita, que consiste en una alteración de la fusión de los tejidos que formarán labio superior y paladar duro y blando en la etapa embrionaria. En cuanto a su prevalencia, la fisura labiopalatina asociada o no a un síndrome, es considerada un problema de salud pública, ya que corresponde a la malformación más común a nivel mundial, afectando a 1 de cada 1.200 nacidos vivos (Bender, 2000).

Aproximadamente un $70 \%$ de los casos de fisuras labiopalatinas corresponden al tipo no sindrómico (FLPNS). Los pacientes con FLPNS, frecuentemente presentan deficiencia tridimensional en el desarrollo maxilar, principalmente producto de las cicatrices ocasionadas por las múltiples cirugías a las que son sometidos. Esta deficiencia puede estar influenciada por el patrón de crecimiento facial heredado. La hipoplasia maxilar contribuirá a generar una morfología facial anormal, produciendo en ellos una apariencia clínica característica: perfil cóncavo; inadecuado soporte para la proyección anterior del labio superior y de la punta de la nariz; visualización disminuida de los incisivos superiores; mordida cruzada y/o mordida invertida (Shahrokh et al.). 
GUERRERO, L. M. T.; TRAUB, V. V.; ZURSIEDEL, P. M. I.; ÁLAMOS, S. C.; NOGUERA, P. A.; GUTIÉRREZ, M. C. \& SOLÉ, V. P. Cambios faciales y su estabilidad en el tiempo en pacientes con fisura labiopalatina no sindrómica sometidos a distracción osteogénica maxilar con distractor externo rígido. Revisión narrativa. Int. J. Odontostomat., 13(2):123-131, 2019.

Actualmente, existen dos alternativas para la corrección quirúrgica de la hipoplasia maxilar en pacientes con FLPNS: la cirugía ortognática y la distracción osteogénica del tercio medio. A pesar de que la cirugía ortognática es la técnica más comúnmente empleada desde la década de los 70 , desde el año 1990 que la distracción osteogénica es reconocida también como una alternativa aceptable. La distracción osteogénica consiste en inducir la formación de tejido óseo utilizando los mecanismos naturales de reparación de éste. Se realiza una osteotomía que es seguida por la separación gradual de los fragmentos, produciéndose una osificación en el lugar de la distracción por una inducción mesenquimática de células osteoprogenitoras en el área de distracción (Sahoo et al., 2014). A pesar de que la distracción osteogénica maxilar ofrece una alternativa viable en la que es posible abordar sólo el maxilar hipoplásico, han habido reportes que cuestionan la eficacia de la técnica, debido a la posible recidiva de ésta.

El objetivo de esta revisión es describir el uso de la distracción osteogénica maxilar con distractor externo rígido o RED (por su denominación en inglés, "Rigid External Distractor") en el tratamiento de la retrusión del tercio medio en pacientes con FLPNS. Asimismo, se describirán los resultados obtenidos por este procedimiento y la estabilidad en el tiempo de dicha técnica quirúrgica.

\section{MATERIAL Y MÉTODO}

Se realizó una búsqueda de la evidencia disponible en las siguientes bases de datos: PubMed, EBSCO, BEIC, Cochrane Library y el metabuscador Epistemonikos a través de las palabras clave "cleft palate"; "cleft lip and palate"; "distraction osteogenesis"; osteodistraction"; "callotasis"; "callotases" y "callus distraction", "maxillary hypoplasia"; "midface hypoplasia"; "hypoplastic maxilla"; "maxillary deficiency"; "retromaxilla" y "maxillary retrognatism" relacionadas entre sí con los términos booleanos AND y OR.

De los artículos obtenidos, se filtró por título, resumen y lectura a texto completo. Se seleccionaron aquellos que cumplían con los siguientes criterios de inclusión: artículos relativos a la pregunta de investigación, estudios in vivo, sin límite de año de publicación ni idioma, disponibles en texto com- pleto. Fueron excluidas aquellos que hacen referencia a pacientes con FLP sindrómica, utilización de distracción osteogénica en huesos distintos al maxilar, expansión maxilar con distracción osteogénica, utilización de dispositivos distintos al RED, estudios animales y opiniones de expertos. Se evaluó el nivel de evidencia científica y el grado de recomendación de los artículos con las pautas del Centre for Evidence-Based Medicine de la Universidad de Oxford. Para evaluar la calidad de reporte de los artículos seleccionados se utilizaron las pautas PRISMA, CONSORT, STROBE y MINCIR-EOD. Para evaluar el riesgo de sesgo de los ensayos clínicos seleccionados se utilizó una modificación de la pauta propuesta por The Cochrane Collaboration y se le asignó un puntaje a cada artículo. Por último, para evaluar la ética de los artículos se consideró la existencia de consentimiento informado, la aprobación de un comité de ética y la ausencia de conflictos de interés.

\section{RESULTADOS Y DISCUSIÓN}

Luego de haber realizado la búsqueda electrónica con las estrategias mencionadas anteriormente y tras aplicar los criterios de inclusión y exclusión, 20 artículos fueron seleccionados para ser incluidos en la revisión narrativa. (Fig. 1).
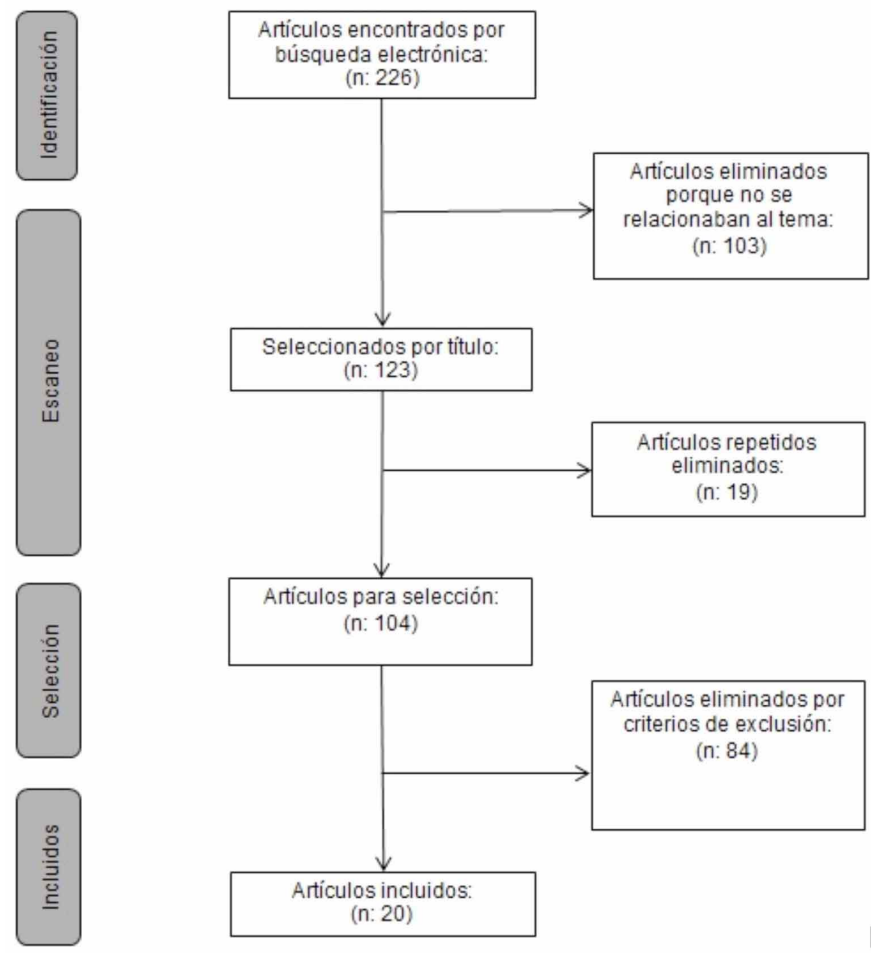

Fig. 1. Flujograma de selección de artículos. 
GUERRERO, L. M. T.; TRAUB, V. V.; ZURSIEDEL, P. M. I.; ÁLAMOS, S. C.; NOGUERA, P. A.; GUTIÉRREZ, M. C. \& SOLÉ, V. P. Cambios faciales y su estabilidad en el tiempo en pacientes con fisura labiopalatina no sindrómica sometidos a distracción osteogénica maxilar con distractor externo rígido. Revisión narrativa. Int. J. Odontostomat., 13(2):123-131, 2019.

Tabla I. Artículos seleccionados.

\begin{tabular}{lcccc}
\hline Autor & Año & Pauta Utilizada & $\begin{array}{c}\text { Porcentaje de } \\
\text { Cumplimiento }\end{array}$ & $\begin{array}{c}\text { Interpretación Calidad } \\
\text { de Reporte }\end{array}$ \\
\hline Saltaji et al. & 2012 & PRISMA & $59 \%$ & $\begin{array}{c}\text { Regular } \\
\text { Mala }\end{array}$ \\
Scolozzi et al. & 2008 & PRISMA & $37 \%$ & Regular \\
Choi et al. & 2012 & CONSORT & $57 \%$ & Regular \\
Daimaruya et al. & 2010 & CONSORT & $51 \%$ & Regular \\
Harada et al. & 2002 & CONSORT & $43 \%$ & Buena \\
Dua et al. & 2014 & MINCIR- EOD & $68 \%$ & Muy Buena \\
Chen et al. & 2011 & MINCIR- EOD & $89 \%$ & Buena \\
Sahoo et al. & 2014 & MINCIR- EOD & $74 \%$ & Regular \\
Suzuki \& Suzuki & 2007 & MINCIR- EOD & $53 \%$ & Regular \\
Minami et al. & 2007 & MINCIR- EOD & $53 \%$ & Buena \\
Cho et al. & 2006 & MINCIR- EOD & $79 \%$ & Regular \\
Rachmiel et al. & 2006 & MINCIR- EOD & $53 \%$ & Buena \\
Yamauchi et al. & 2006 & MINCIR- EOD & $68 \%$ & Muy Buena \\
Suzuki et al. & 2004 & MINCIR- EOD & $84 \%$ & Buena \\
Swennen et al. & 2000 & MINCIR- EOD & $63 \%$ & Regular \\
Aksu et al. & 2010 & MINCIR- EOD & $58 \%$ & Regular \\
Wen-Ching Ko et al. & 2000 & MINCIR- EOD & $58 \%$ & Buena \\
Gürsoy et al. & 2010 & MINCIR- EOD & $63 \%$ & Regular \\
Harada et al. & 2006 & MINCIR- EOD & $42 \%$ & Regular \\
Kim et al. & 2015 & STROBE & $50 \%$ &
\end{tabular}

Estos corresponden a 2 revisiones sistemáticas, 3 ensayos clínicos, 14 estudios observacionales descriptivos y 1 estudio observacional analítico (Tabla I).

Uso de la distracción osteogénica maxilar con RED en pacientes con FLPNS: En general, las principales indicaciones de la distracción osteogénica maxilar en pacientes con fisura son la presencia de hipoplasia maxilar severa e insuficiencia velofaríngea. El promedio de edad de los pacientes tratados con distracción osteogénica en los artículos analizados varía entre los 11,1 años y 21,56 años (Sahoo et al.; Swennen et al., 2000a,b; Harada et al., 2002; Suzuki et al., 2004; Cho \& Kyung, 2006; Harada et al., 2006; Yamauchi et al., 2006; Suzuki \& Suzuki, 2007; Minami et al., 2007; Aksu et al., 2010; Daimaruya et al., 2010; Gürsoy et al., 2010; Chen et al., 2011; Choi et al., 2012; Dua et al., 2014; Kim et al., 2015). Se excluyen dos de los artículos incluidos en este trabajo, ya que estos sólo mencionan el intervalo de edad de los pacientes tratados con distracción osteogénica (5,2 a 25,7 años) y no la media de ésta (Wen-Ching Ko et al., 2000; Rachmiel et al., 2006). A pesar de ello, los resultados encontrados concuerdan con lo descrito como una ventaja de la distracción osteogénica, un procedimiento que puede ser realizado a cualquier edad, a diferencia del tratamiento mediante tratamiento quirúrgico convencional, retrasando la edad de la cirugía hasta que el paciente alcance la madurez ósea esqueletal (Cho \& Kyung).
En cuanto al uso de la distracción osteogénica con RED; se encontró que la osteotomía más utilizada fue la tipo Le Fort I alta (Sahoo et al.; Aksu et al.; Dua et al.; Choi et al.; Minami et al.; Swennen et al., 2000a,b; Harada et al., 2002; Gürsoy et al.; Harada et al., 2006; Wen-Ching Ko et al.), seguida por la tipo Le Fort I clásica (Suzuki et al.; Chen et al.; Daimaruya et al.; Suzuki \& Suzuki; Yamauchi et al.; Kim et al.). Algunos estudios, por otro lado, mencionan haber realizado la distracción osteogénica tanto con Le Fort I clásica, como con Le Fort I alta, según las condiciones de cada paciente, para evitar dañar los gérmenes dentarios (Cho \& Kyung; Rachmiel et al., 2006).

Después de un período de latencia que variaba entre dos a cinco días, la activación comenzaba con una tasa de $1 \mathrm{~mm}$ cada 24 horas, generalmente con 2 activaciones al día (Dua et al.; Choi et al.; Daimaruya et al.; Minami et al.; Swennen et al., 2000a,b; Harada et al., 2002; Gürsoy et al.; Harada et al., 2006; Rachmiel et al., 2006; Wen-Ching Ko et al.); existiendo también estudios con una activación al día (Susuki et al , 2004; Aksu et al.; Cho \& Kyung; Chen et al.; Susuki et al., 2007) y otros con 4 activaciones diarias (Sahoo et al.). Sólo dos artículos realizaron la distracción osteogénica con una tasa de activación mayor a $1 \mathrm{~mm}$ diario; el estudio de Yamauchi et al., realizaba una activación del distractor de 1 a $2 \mathrm{~mm}$ al día, mientras que el estudio de Kim et al. la realizaba de 1 a $1,5 \mathrm{~mm}$ al día (Yamauchi et al.; Kim et al.). Swennen et al. (2000a,b) 
GUERRERO, L. M. T.; TRAUB, V. V.; ZURSIEDEL, P. M. I.; ÁLAMOS, S. C.; NOGUERA, P. A.; GUTIÉRREZ, M. C. \& SOLÉ, V. P. Cambios faciales y su estabilidad en el tiempo en pacientes con fisura labiopalatina no sindrómica sometidos a distracción osteogénica maxilar con distractor externo rígido. Revisión narrativa. Int. J. Odontostomat., 13(2):123-131, 2019.

indican que el tiempo en que se consigue avanzar el maxilar una misma cantidad de milímetros en dos pacientes distintos, no siempre va a ser el mismo, debido a que el movimiento puede estar influenciado por la angulación del vector de distracción, por falta de rigidez de los alambres de tracción y, en caso de existir, por la flexión de las miniplacas de anclaje esqueletal. Finalmente, terminado el período de activación tras lograr el avance maxilar deseado, el distractor se mantiene en su lugar por un período de consolidación, que va entre las 2 y 12 semanas, dependiendo del estudio.

Los estudios incluidos utilizaron con mayor frecuencia el anclaje del distractor directamente al maxilar, pero se ha descrito que la férula intraoral es el sistema de anclaje más utilizado. En relación al anclaje dentario, a pesar de que existe evidencia controversial al respecto, éste puede resultar en movimientos dentarios indeseados. De los artículos que utilizaron un sistema de anclaje dentario, 3 de ellos reportaron este tipo de movimientos. Suzuki et al. registraron tanto extrusión, como palatinización de los incisivos centrales superiores; así como extrusión y distalización de los primeros molares superiores. Otros reportaron un movimiento hacia anterior de los incisivos centrales superiores y mesialización de los primeros molares superiores (Aksu et al.; Gürsoy et al.).

Para evitar este problema, se introdujo el uso de placas de retención en el sistema de RED. Este método de anclaje posee otras ventajas con respecto a las férulas intraorales, como permitir la transferencia directa de fuerza hacia el hueso. Además, se disminuye el riesgo de dañar el labio superior. Daimaruya et al. afirma que utilizar placas de retención es la mejor opción para obtener un avance maxilar ideal sin perder soporte dentoalveolar y evitar sobrecargar los tejidos periodontales, especialmente en una dirección vertical (Daimaruya et al.). A pesar de las ventajas que brinda este modo de anclaje, su principal problema consiste en la instalación de las placas, ya que implica un procedimiento a veces difícil por ser el maxilar un hueso extremadamente delgado y por el riesgo de dañar alguna raíz dentaria; además, se requiere de una segunda intervención quirúrgica para remover las placas (Scolozzi, 2008).

Cambios esqueletales obtenidos mediante distracción osteogénica maxilar con RED: La cefalometría de perfil es el método más utilizado tanto para la evaluación pre y post operatoria, así como para el seguimiento de los pacientes con FLP sometidos a distracción osteogénica. En esta, los puntos de referencia se identifican no sólo en base a las características de las superficies esqueletales y blandas, sino que también en base a las suturas y a las diferentes radiopacidades (Chen et al.).

A pesar de que una limitación de esta revisión fue la inexistencia de estandarización de los puntos cefalométricos utilizados para evaluar los cambios tanto esqueletales como blandos luego del uso de distracción osteogénica maxilar con sistema RED, los cambios esqueletales encontrados en los estudios pueden dividirse en modificaciones de dos tipos:

Cambios lineales: El promedio del avance maxilar horizontal, generalmente evaluado mediante el cambio de posición del punto $A$, fue de $10 \mathrm{~mm}$ a $15 \mathrm{~mm}$ en la mayoría de los estudios (Sahoo et al.; Suzuki et al.; Dua et al.; Choi et al.; Cho \& Kyung; Chen et al.; Suzuki \& Suzuki; Gürsoy et al.); entre $7,5 \mathrm{~mm}$ y $10 \mathrm{~mm}$ en una menor cantidad de estudios (Aksu et al.; Daimaruya et al.; Swennen et al., 2000a,b; Wen-Ching Ko et al.); y mayor a $15 \mathrm{~mm}$ en sólo tres artículos (Minami et al.; Kim et al.; Rachmiel et al., 2006). Ni el estudio de Yamauchi et al., ni los de Harada et al. mencionan el promedio de avance maxilar obtenido (Yamauchi et al.; Harada et al., 2002, 2006). A pesar de que la mayoría de los estudios sólo describen el avance maxilar en un sentido sagital, aquellos estudios que evaluaron el promedio del movimiento en sentido vertical reportaron que, durante la distracción, el maxilar se movilizó hacia inferior entre $1,6 \mathrm{~mm}$ a 3 $\mathrm{mm}$ en la mayoría de los casos (Suzuki et al.; Dua et al.; Gürsoy et al.), y entre $3 \mathrm{~mm}$ y $7 \mathrm{~mm}$ en dos artículos (Daimaruya et al.; Yamauchi et al.).

Cambios angulares: El aumento del promedio del ángulo SNA varió entre $4,7^{\circ}$ y $13,1^{\circ}$ en los distintos estudios (Sahoo et al.; Dua et al.; Cho \& Kyung; Daimaruya et al.; Suzuki \& Suzuki; Minami et al.; Yamauchi et al.; Kim et al.; Gürsoy et al.; Wen-Ching Ko et al.); mientras que el aumento del promedio del ángulo ANB fluctuó entre los 6,7 $7^{\circ}$ y los $13,3^{\circ}$ (Sahoo et al.; Dua et al.; Choi et al.; Daimaruya et al.; Suzuki \& Suzuki; Yamauchi et al.; Kim et al.; Gürsoy et al.; Wen-Ching Ko et al.). A pesar de que todos indican que la dirección esperada del movimiento maxilar mediante distracción osteogénica es anteroinferior (Saltaji et al. 2012), sólo un estudio realizado por Aksu et al., encontró que el maxilar rotó significativamente en sentido anti horario; movilizándose en una dirección anterosuperior. Los autores de este trabajo, sin embargo, afirman que el punto de aplicación de la fuerza de distracción pudo haber contribuido en el efecto rotacional observado; 
GUERRERO, L. M. T.; TRAUB, V. V.; ZURSIEDEL, P. M. I.; ÁLAMOS, S. C.; NOGUERA, P. A.; GUTIÉRREZ, M. C. \& SOLÉ, V. P. Cambios faciales y su estabilidad en el tiempo en pacientes con fisura labiopalatina no sindrómica sometidos a distracción osteogénica maxilar con distractor externo rígido. Revisión narrativa. Int. J. Odontostomat., 13(2):123-131, 2019.

junto con la falta de un injerto óseo alveolar, ya que esto último provoca la existencia de una discontinuidad entre los segmentos óseos (Aksu et al.).

En relación a los cambios en la mandíbula posteriores a la distracción osteogénica maxilar, han sido evidenciadas rotaciones mandibulares significativas, que van de acuerdo al grado de avance maxilar alcanzado, como resultado de un aumento en la altura facial debido al movimiento inferior del maxilar en estos pacientes. De losartículos analizados, 5 reportaron rotaciones mandibulares en sentido horario, reflejado cefalométricamente como aumento en la divergencia entre maxilar y mandíbula, y clínicamente como una tendencia a abrirse la mordida. Sin embargo, sólo en el estudio realizado por Suzuki et al., las direcciones de estas rotaciones no fueron uniformes: durante el período de distracción, un $66,6 \%$ de los pacientes presentó una rotación mandibular en sentido horario, y un 33,3\% en un sentido anti horario (Suzuki et al.; Yamauchi et al.; Gürsoy et al.; Rachmiel et al., 2006; Wen-Ching Ko et al.). Este último estudio, además, fue el único que realizó un análisis cuantitativo para examinar los efectos mandibulares que producen los cambios dentoesqueletales del maxilar distraído. Finalmente, en base a este análisis, se identificó la existencia de una relación significativa entre los dientes y las estructuras óseas, concluyendo que el cambio posicional vertical de los incisivos centrales superiores podría determinar la dirección de rotación mandibular (Suzuki et al.).

Cambios en tejidos blandos obtenidos mediante distracción osteogénica maxilar con RED: Pocos estudios han sido publicados en donde evalúen los cambios en los tejidos blandos de pacientes fisurados tras la distracción osteogénica. Sin embargo, es de gran importancia para el cirujano, en la planificación de los movimientos maxilares quirúrgicos de los pacientes con FLP, el comprender los cambios que produce este procedimiento en los tejidos blandos de la cara. Los cambios significativos en los tejidos blandos, producidos por la distracción osteogénica maxilar con sistema RED, se ubican principalmente en la nariz y labio superior. Según los estudios, los efectos de esta técnica sobre la región nasal incluyen un aumento tanto del ángulo nasolabial (Sahoo et al.; Dua et al.; Harada et al., 2002; Rachmiel et al., 2006; Wen-Ching Ko et al.), como de la proyección de la punta de la nariz (Daimaruya et al.; Wen-Ching Ko et al.) y el punto subnasal (Harada et al., 2002; Daimaruya et al.). Específicamente, el aumento del promedio del ángulo nasolabial reportado fue entre $5^{\circ}$ y $23,1^{\circ}$. Además, un estudio señala que la punta nasal se moviliza hacia superior después de la distracción (Wen-Ching Ko et al.). En cuanto a la relación entre el movimiento sagital de la punta de la nariz y la espina nasal anterior, WenChing Ko et al. y Harada et al., encontraron que ésta era de 0,53:1 y 0,56:1 respectivamente (Harada et al., 2002; Wen-Ching Ko et al.).

Los cambios producidos en la zona del labio superior corresponden al avance horizontal y descenso del labio superior (Sahoo et al.; Harada et al., 2002; Rachmiel et al., 2006; Daimaruya et al.); cuyo movimiento se relaciona con el de la espina nasal anterior y el margen incisal de los incisivos centrales superiores en una razón de 0,7:1, y entre 0,6:1 y 0,8:1, respectivamente (Harada et al., 2002; Wen-Ching Ko et al.). Por otro lado, Wen-Ching Ko et al., reportó también una disminución de la curvatura y el espesor del labio superior; así como también un aumento de la curvatura del labio inferior (Wen-Ching Ko et al.).

Es necesario mencionar el efecto que este tratamiento podría provocar en el cierre velofaríngeo, el que se ve comprometido luego de avances maxilares convencionales mayores a $10 \mathrm{~mm}$, lo que crea una gran brecha entre el paladar y la pared faríngea, empeorando así la función velo faríngea y fomentando la hipernasalidad (Scolozzi). Una de las ventajas que se han descrito en relación a la distracción osteogénica, es que el movimiento progresivo del maxilar favorece la adaptación de las estructuras velofaríngeas, dado que el paladar es avanzado en pequeños incrementos (Scolozzi). En contraste con lo anterior, existen estudios que afirman que este tratamiento puede, de igual forma, producir incompetencia velofaríngea e hipernasalidad (lida et al., 2007). Chanchareonsook et al. (2007) afirma que el impacto del avance maxilar mediante distracción osteogénica maxilar en el habla y en el estado velofaríngeo es variable. Este último autor, además sostiene que la máxima cantidad de avance maxilar reportada más allá de la cual es altamente probable que ocurra un deterioro de la función velofaríngea, es de $15 \mathrm{~mm}$ para la distracción osteogénica y de 10 $\mathrm{mm}$ para las osteotomías convencionales.

En una revisión sistemática realizada el año 2008 por Scolozzi, se indica que el resultado más comúnmenteencontrado luego de la distracción osteogénica fue una función velofaríngea sin cambios, independientemente de la magnitud del avance maxilar. El empeoramiento de la hipernasalidad y de la fonoarticulación varió de $11 \%$ a $17 \%$, y de $6 \%$ a 14 $\%$, respectivamente; mientras que un $70 \%$ de casos 
GUERRERO, L. M. T.; TRAUB, V. V.; ZURSIEDEL, P. M. I.; ÁLAMOS, S. C.; NOGUERA, P. A.; GUTIÉRREZ, M. C. \& SoLÉ, V. P. Cambios faciales y su estabilidad en el tiempo en pacientes con fisura labiopalatina no sindrómica sometidos a distracción osteogénica maxilar con distractor externo rígido. Revisión narrativa. Int. J. Odontostomat., 13(2):123-131, 2019.

reportó una mejora en la fonoarticulación. También se encontró que el deterioro de la hipernasalidad estaba relacionado con la cantidad de avance maxilar obtenido mediante la distracción, especialmente en pacientes que no habían sido sometidos a faringoplastía previa. Además, se encontró que los siguientes cambios anatómicos permiten una mejor función y habla: aumento del espacio nasofaríngeo, ya que favorece la respiración nasal; verticalización del septum nasal; mejora de la función de la tuba auditiva; aumento del espacio oral y mejora en las relaciones dentales y en la oclusión. Por último, en relación a los cambios que produce la distracción osteogénica en el habla, las mejoras más importantes se encontraron en aquellos fonemas en que la lengua se posiciona en el paladar o el margen alveolar (Scolozzi).

Recidiva de los cambios obtenidos mediante distracción osteogénica maxilar con RED: Los estudios reportaron un movimiento esqueletal estable tras el uso de la distracción osteogénica debido a la interposición de tejido óseo nuevo en la región pterigomaxilar. Esta técnica, en la mayoría de los casos, presenta un porcentaje de recidiva en los cambios que provoca. Es importante señalar que, debido a que todos los estudios utilizaban distintos parámetros faciales para evaluar la estabilidad que se logra con la distracción osteogénica y que estos parámetros cefalométricos eran registrados en distintos momentos post distracción, dependiendo del estudio analizado; es difícil realizar una comparación e interpretación entre las recidivas encontradas en los diferentes artículos. Además, la mayoría de los artículos incluidos que evaluaban la estabilidad de los cambios obtenidos mediante distracción osteogénica, carecían de detalles cuantitativos en relación a ésta; lo cual también constituyó una limitación al momento de realizar la revisión. Para propósitos de esta revisión, cualquier cambio del maxilar en una dirección superior o posterior, luego de la distracción osteogénica, fue considerada como recidiva. La razón de esto se basa en lo que ya se ha sido mencionado con anterioridad: la dirección del movimiento del maxilar que se espera luego del tratamiento de la retrusión del tercio medio con distracción osteogénica generalmente corresponde a anterior e inferior.

Los estudios incluidos mostraron una recidiva en sentido horizontal que, a los 12 meses, fue entre $20 \%$ y $25 \%$ en la mayoría de los casos (Suzuki et al.; Choi et al.; Cho \& Kyung), menos de $15 \%$ en sólo un artículo (Daimaruya et al.) y entre $15 \%$ y $20 \%$ en otro artículo (Dua et al.). De los estudios que evaluaban la estabilidad de los cambios esqueletales luego de la distracción, sólo uno reportó un maxilar estable en el sentido horizontal sin recidiva a los 15 - 16 meses de seguimiento de los pacientes incluidos (Swennen et al., 2000a,b). Esto último concuerda con lo publicado por Rachmiel et al., el año 2005, quienes tras realizar un avance maxilar promedio de $21 \mathrm{~mm}$ en 12 pacientes y un seguimiento por dos años, no reportaron cambios estadísticamente significativos en cuanto a la posición del maxilar (Rachmiel et al., 2005). Por otro lado, de aquellos estudios que evaluaron también el movimiento del maxilar en un sentido vertical: dos no reportaron recidiva hacia superior (Sahoo et al.; Daimaruya et al.), mientras que otros autores sí encontraron una recidiva significativa en esta dirección (Suzuki et al.).

Las principales causas de recidiva reportadas se han relacionado con avances mayores a $10 \mathrm{~mm}$, cicatrices presentes en los pacientes con FLP y edad en que se realizó la distracción osteogénica; ya que a pesar de que el crecimiento del maxilar pudo haber terminado, la mandíbula todavía puede conservar un potencial de crecimiento (Dua et al.).

En relación a la recidiva que se observa en pacientes en crecimiento sometidos a distracción osteogénica maxilar, Chen et al., tras someter a 12 pacientes en crecimiento, con un promedio de edad de 11,12 años y con hipoplasia maxilar severa, encontraron una recidiva importante al año de seguimiento, y una recurrencia de la deformidad facial a los cinco años de seguimiento, lo que se debió principalmente al crecimiento relativo existente entre el segmento superior e inferior de la cara. Es por esto que sugiere la necesidad de predecir el futuro crecimiento de la mandíbula, para un mejor resultado quirúrgico; lo cual es complejo en pacientes con retrusión del tercio medio, ya que es sabido que pacientes que presentan una maloclusión clase III tienen una tendencia a aumentar y prolongar su crecimiento mandibular. Resultados similares se observaron en tres pacientes con dentición mixta incluidos en un estudio realizado por Cho \& Kyung. Luego de someter a distracción osteogénica con RED a pacientes, tanto en crecimiento como adultos, se encontró la presencia de una mayor "recaída" del ángulo ANB en tres sujetos con dentición mixta. Debido a ello, se concluyó que el maxilar sometido a distracción osteogénica tiene una tasa de crecimiento más baja que la mandíbula en pacientes en crecimiento. Por lo tanto la tasa de recidiva de la retrusión maxilar en pacientes con FLP en crecimiento, es realmente debido al continuo crecimiento de la mandíbula, la que presenta un mayor potencial de crecimiento que el maxilar (Cho \& Kyung). 
GUERRERO, L. M. T.; TRAUB, V. V.; ZURSIEDEL, P. M. I.; ÁLAMOS, S. C.; NOGUERA, P. A.; GUTIÉRREZ, M. C. \& SOLÉ, V. P. Cambios faciales y su estabilidad en el tiempo en pacientes con fisura labiopalatina no sindrómica sometidos a distracción osteogénica maxilar con distractor externo rígido. Revisión narrativa. Int. J. Odontostomat., 13(2):123-131, 2019.

Otros autores recomiendan realizar una sobre corrección en caso de realizar el avance en períodos de crecimiento. Esto último, para compensar aún más el crecimiento de los tejidos circundantes, especialmente la mandíbula y de esta manera compensar la recidiva y recurrencia de la retrusión (Dua et al.; Harada et al., 2006). En base a eso, es necesario recalcar que la incapacidad para predecir el futuro crecimiento de las estructuras anatómicas asociadas al maxilar es crucial durante la planificación preoperatoria (Dua et al.), ya que a pesar de que el crecimiento sagital del maxilar cesa entre los 12 y 14 años de edad, la mandíbula continúa creciendo por más tiempo, debido a un gradiente céfalo caudal (Sahoo et al.).

Otra causa de recidiva, podría ser el movimiento dentario producido por el uso de aquellos RED con anclaje dentario. En la revisión sistemática realizada por Scolozzi el año 2008, se reportó que cuando las fuerzas de distracción maxilar son entregadas al hueso mediante anclaje dentario, pueden ocurrir compensaciones dentarias indeseadas como proinclinación y/ o extrusión de los incisivos maxilares y comprometiendo la estabilidad, dada la alta propensión del movimiento dentario a la recidiva (Scolozzi). En efecto, uno de los artículos que reportó cambios dentarios luego de la distracción osteogénica con RED, y que posteriormente evaluó la mantención de estos a largo plazo, menciona la existencia de una recidiva significativa de éstos durante los primeros seis meses posteriores a la distracción (Suzuki et al.; Rachmiel et al., 2006). Por lo tanto, a pesar de que hay quienes han dicho que los movimientos dentarios producidos por el anclaje dentario generalmente no son estadísticamente significativos; en caso de existir, éstos pueden ser responsables de una recidiva dentoesqueletal postoperatoria significativa en muchos casos (Minami et al.; Swennen et al., 2000a,b).

El tiempo de consolidación, por otro lado, también podría estar relacionado con la recidiva de la hipoplasia maxilar. Cho \& Kyung sugieren que, para disminuir la tasa de recidiva, es necesaria la mantención del RED por un período mayor a seis semanas o utilizar una máscara facial de retención por esta misma cantidad de tiempo o más. Rachmiel et al., luego de demostrar que los dispositivos de distracción externa elongan el maxilar de manera exitosa y estable tras someter a 18 pacientes a distracción osteogénica, con un período de consolidación de 8 semanas y evaluar los cambios obtenidos por un período de dos años, afirmaron que es importante utilizar largos períodos de consolidación para lograr resultados estables y prevenir recidivas
(Rachmiel et al., 2006). Suzuki et al. tras realizar un análisis cuantitativo en relación a los factores que afectan la estabilidad del maxilar después de la distracción osteogénica, encontraron que, en la dimensión vertical, la recidiva maxilar estaba significativamente relacionada con la cantidad de movimiento quirúrgico maxilar; mientras que la recidiva maxilar horizontal estaba significativamente relacionada con el grado de hipoplasia maxilar existente previo a la cirugía. Esto último se debe a que la severidad de la hipoplasia está fuertemente relacionada con las tensiones de las cicatrices y de los tejidos blandos, las cuales a su vez han sido identificadas como el principal factor causal de la recidiva maxilar en este sentido (Suzuki et al.).

En relación al momento en el cual se produce la recidiva; se encontró que la recidiva dentoesqueletal significativa ocurre dentro de los primeros 6 meses después del procedimiento. En base a esto, concuerdan en que la estabilidad dentoesqueletal se alcanza a los 6 meses post cirugía (Suzuki et al.; Cho \& Kyung) $y$, por lo mismo, sugiere la realización de una sobre corrección de 20 - $30 \%$, dependiendo de la severidad de la deficiencia e independientemente de la edad de paciente sometido a la distracción, con el fin de prevenir que la recidiva sea notoria (Cho \& Kyung). Aksu et al., tras someter a 7 individuos adultos a distracción osteogénica maxilar, reportó una recidiva de $28,7 \%$ a los 37,7 meses. Debido a que en este último artículo, todos los pacientes habían finalizado su crecimiento, probablemente la recidiva se debió principalmente a la fuerte resistencia que ejercen los tejidos blandos como resultados de las cicatrices, más que al déficit de crecimiento que presenta el maxilar fisurado (Aksu et al.). Gürsoy et al., luego de realizar un seguimiento de 5 años a pacientes con FLP, encontraron una recidiva maxilar del $23,9 \%$. Sin embargo, recalca que a pesar de que el maxilar se movilizó significativamente hacia posterior durante los cinco años consecutivos a la distracción osteogénica; las evaluaciones clínicas y cefalométricas realizadas al finalizar el período de seguimiento indican que éstos aún tienen clase I esqueletal, y que las mejorías faciales se habían mantenido desde el procedimiento quirúrgico (Gürsoy et al.).

En cuanto a la estabilidad que proporciona la distracción osteogénica al cambio en los tejidos blandos, Wen-Ching Ko et al. encontraron que, en un período promedio de 14,4 meses de seguimiento, la convexidad facial que había aumentado $5,59^{\circ}$ durante la distracción, disminuyó $2,96^{\circ}$ en este tiempo debido al movimiento del maxilar hacia posterior y al movimiento 
GUERRERO, L. M. T.; TRAUB, V. V.; ZURSIEDEL, P. M. I.; ÁLAMOS, S. C.; NOGUERA, P. A.; GUTIÉRREZ, M. C. \& SOLÉ, V. P. Cambios faciales y su estabilidad en el tiempo en pacientes con fisura labiopalatina no sindrómica sometidos a distracción osteogénica maxilar con distractor externo rígido. Revisión narrativa. Int. J. Odontostomat., 13(2):123-131, 2019.

de la mandíbula y de los incisivos inferiores. Lo mismo sucedió con otros parámetros evaluados, los cuales mostraron una tendencia a moverse hacia su posición original: el labio superior se movilizó hacia posterior, el espesor del labio inferior disminuyó, y con esto la curvatura de éste se aplanó, restaurando el contorno que poseía antes de la intervención. Todos los parámetros cefalométricos relacionados con la región nasal, así como el margen bermellón del labio inferior, se mostraron estables durante el seguimiento. Sin embargo, a pesar de que algunos parámetros del perfil blando evaluados sí tendieron a movilizarse hacia su posición original, los autores indican que el tratamiento fue estable al año de seguimiento (Wen-Ching Ko et al.).

Corregir la deficiencia maxilar por FLP constituye un desafío para el cirujano. La distracción osteogénica maxilar es una alternativa prometedora para el tratamiento de esta. Las principales indicaciones que tiene corresponden a casos de hipoplasia maxilar severa y/o insuficiencia velofaríngea. Ya que este procedimiento puede ser realizado en pacientes en crecimiento, corregir tempranamente la desarmonía facial puede considerarse como otra indicación.

Es posible concluir que la distracción osteogénica maxilar con RED, se trata de una alternativa terapéutica eficaz en el manejo de la hipoplasia maxilar en pacientes con FLPNS, los cuales requieren una evaluación cuidadosa, ya que este tratamiento no asegura la no ocurrencia de efectos negativos sobre el cierre velofaríngeo.

GUERRERO, L. M. T.; TRAUB, V. V.; ZURSIEDEL, P. M. I.; ÁlAMOS, S. C.; NOGUERA, P. A.; GUTIÉRREZ, M. C. \& SOLÉ, V. P. Facial changes and their stability over time in non-syndromic cleft lip and palate patients undergoing maxillary distraction osteogenesis through rigid external distractor. Narrative review. Int. J. Odontostomat., 13(2):123131, 2019.

ABSTRACT: Cleft lip and palate is the world's most prevalent head and neck malformation, within which, nonsyndromic is the most frequent. Patients with this malformation in many cases present maxillary hypoplasia. Maxillary distraction osteogenesis through a rigid external distractor constitutes an alternative to correct this skeletal deficiency. The objective of this study was to describe facial changes and their stability over time in non-syndromic cleft lip and palate patients undergoing distraction osteogenesis through rigid external distractor. An electronic search was carried out in PubMed database, Epistemonikos, EBSCO, BEIC and The Cochrane Library through the keywords cleft palate; cleft lip and palate; distraction osteogenesis; osteodistraction; callotasis; callotases; callus distraction, maxillary hypoplasia; midface hypoplasia; hypoplastic maxilla; maxillary deficiency; retromaxilla and maxillary retrognatism, related to each other with the Boolean terms AND and OR. For this analysis 20 articles were selected: 2 systematic reviews, 3 clinical trials, 14 descriptive observational studies and 1 analytic observational study. Maxillary distraction osteogenesis through rigid external distractor is an effective alternative in the treatment of midface retrusion in non-syndromic cleft lip and palate patients. However, this technique does not completely achieve stable changes, due to multiple factors related to its recurrence.

KEY WORDS: distraction osteogenesis, cleft lip and palate, Le Fort osteotomy, orthognathic surgery.

\section{REFERENCIAS BIBLIOGRÁFICAS}

Aksu, M.; Saglam-Aydinatay, B.; Akcan, C. A.; El, H.; Taner, T.; Kocadereli, I.; Tuncbilek, G. \& Mavili, M. E. Skeletal and dental stability after maxillary distraction with a rigid external device in adult cleft lip and palate patients. J. Oral Maxillofac. Surg., 68(2):254-9, 2010.

Bender, P. L. Genetics of cleft lip and palate. J. Pediatr. Nurs., 15(4):242-9, 2000.

Chanchareonsook, N.; Whitehill, T. \& Samman, N. Speech outcome and velopharyngeal function in cleft palate: comparison of Le Fort I maxillary osteotomy and distraction osteogenesis-early results. Cleft Palate Craniofac. J., 44(1):23-32, 2007.

Chen, P. K.; Por, Y. C.; Liou, E. J. \& Chang, F. C. Maxillary distraction osteogenesis in the adolescent cleft patient: three-dimensional computed tomography analysis of linear and volumetric changes over five years. Cleft Palate Craniofac. J., 48(4):445-54, 2011.

Cho, B. C. \& Kyung, H. M. Distraction osteogenesis of the hypoplastic midface using a rigid external distraction system: the results of a one- to six-year follow-up. Plast. Reconstr. Surg., 118(5):120112, 2006

Choi, H. Y.; Hwang, C. J.; Kim, H. J.; Yu, H. S. \& Cha, J. Y. Maxillary anterior segmental distraction osteogenesis with 2 different types of distractors. J. Craniofac. Surg., 23(3):706-11, 2012.

Daimaruya, T.; Imai, Y.; Kochi, S.; Tachi, M. \& Takano-Yamamoto, T. Midfacial changes through distraction osteogenesis using a rigid external distraction system with retention plates in cleft lip and palate patients. J. Oral Maxillofac. Surg., 68(7):1480-6, 2010.

Dua, G.; Navin Kumar, A.; Roy, I. D. \& Roy, S. K. Maxillary distraction osteogenesis in cleft lip and palate cases with midface hypoplasia using rigid external distractor: an alternative technique. J. Craniofac. Surg., 25(3):746-51, 2014

Gürsoy, S.; Hukki, J. \& Hurmerinta, K. Five-year follow-up of maxillary distraction osteogenesis on the dentofacial structures of children with cleft lip and palate. J. Oral Maxillofac. Surg., 68(4):744-50, 2010.

Harada, K.; Baba, Y.; Ohyama, K. \& Omura, K. Soft tissue profile changes of the midface in patients with cleft lip and palate following maxillary distraction osteogenesis: a preliminary study. Oral Surg. Oral Med. Oral Pathol. Oral Radiol. Endod., 94(6):6737, 2002.

Harada, K.; Sato, M. \& Omura, K. Long-term maxillomandibular skeletal and dental changes in children with cleft lip and palate after maxillary distraction. Oral Surg. Oral Med. Oral Pathol. Oral Radiol. Endod., 102(3):292-9, 2006. 
GUERRERO, L. M. T.; TRAUB, V. V.; ZURSIEDEL, P. M. I.; ÁLAMOS, S. C.; NOGUERA, P. A.; GUTIÉRREZ, M. C. \& SOLÉ, V. P. Cambios faciales y su estabilidad en el tiempo en pacientes con fisura labiopalatina no sindrómica sometidos a distracción osteogénica maxilar con distractor externo rígido. Revisión narrativa. Int. J. Odontostomat., 13(2):123-131, 2019.

lida, S.; Yagi, T.; Yamashiro, T.; Okura, M.; Takada, K. \& Kogo, M. Maxillary anterior segmental distraction osteogenesis with the dynaform system for severe maxillary retrusion in cleft lip and palate. Plast. Reconstr. Surg., 120(2):508-16, 2007.

Kim, J.; Uhm, K. I.; Shin, D.; Lee, J. \& Choi, H. Maxillary Distraction osteogenesis using a rigid external distractor: which clinical factors are related with relapse? J. Craniofac. Surg., 26(4):117881, 2015.

Minami, K.; Mori, Y.; Tae-Geon, K.; Shimizu, H.; Ohtani, M. \& Yura, Y. Maxillary distraction osteogenesis in cleft lip and palate patients with skeletal anchorage. Cleft Palate Craniofac. J., 44(2):13741, 2007.

Rachmiel, A.; Aizenbud, D. \& Peled, M. Distraction osteogenesis in maxillary deficiency using a rigid external distraction device. Plast. Reconstr. Surg., 117(7):2399-406, 2006.

Rachmiel, A.; Aizenbud, D. \& Peled, M. Long-term results in maxillary deficiency using intraoral devices. Int. J. Oral Maxillofac. Surg., 34(5):473-9, 2005.

Sahoo, N.; Desai, A. P.; Jayan, B. \& Gupta, V. Evaluation of treatment outcome in cleft maxillary hypoplasia treated by rigid external distractor: a cephalometric study. J. Craniofac. Surg., 25(1):1438, 2014.

Saltaji, H.; Major, M. P.; Altalibi, M.; Youssef, M. \& Flores-Mir, C. Long-term skeletal stability after maxillary advancement with distraction osteogenesis in cleft lip and palate patients. Angle Orthod., 82(6):1115-22, 2012.

Scolozzi, P. Distraction osteogenesis in the management of severe maxillary hypoplasia in cleft lip and palate patients. J. Craniofac. Surg., 19(5):1199-214, 2008.

Suzuki, E. Y. \& Suzuki, B. Removable splint with locking attachments for maxillary distraction osteogenesis with the RED system. Int. J. Oral Maxillofac. Surg., 36(12):1153-7, 2007.

Suzuki, E. Y.; Motohashi, N. \& Ohyama, K. Longitudinal dentoskeletal changes in UCLP patients following maxillary distraction osteogenesis using RED system. J. Med. Dent. Sci., 51(1):2733, 2004.

Swennen, G.; Dujardin, T.; Goris, A.; De Mey, A. \& Malevez C. Maxillary distraction osteogenesis: a method with skeletal anchorage. J. Craniofac. Surg., 11(2):120-7, 2000a.

Swennen, G.; Figueroa, A. A.; Schierle, H.; Polley, J. W. \& Malevez, C. Maxillary distraction osteogenesis: a two-dimensional mathematical model. J. Craniofac. Surg., 11(4):312-7, $2000 \mathrm{~b}$.

Wen-Ching Ko, E.; Figueroa, A. A. \& Polley, J. W. Soft tissue profile changes after maxillary advancement with distraction osteogenesis by use of a rigid external distraction device: a 1year follow-up. J. Oral Maxillofac. Surg., 58(9):959-69, 2000.

Yamauchi, K.; Mitsugi, M. \& Takahashi, T. Maxillary distraction osteogenesis using Le Fort I osteotomy without intraoperative down-fracture. Int. J. Oral Maxillofac. Surg., 35(6):493-8, 2006.

\author{
Dirección para correspondencia: \\ Vicente Traub Valdés \\ Universidad de los Andes \\ Monseñor Álvaro del Portillo 12455 \\ Las Condes - Santiago \\ CHILE
}

Email: vatraub@miuandes.cl

Recibido : 03-08-2018

Aceptado: 18-10-2018 